\title{
Rastreio do consumo de álcool nos cuidados de saúde primários - atitudes dos utentes
}

Pedro Pereira Campos, ${ }^{*}$ Cristina Ribeiro**

\section{RESUMO}

Objectivos: Caracterizar o consumo de álcool dos utentes dos Cuidados de Saúde Primários (CSP) com idade igual ou superior a 16 anos; determinar as atitudes dos participantes face ao rastreio do consumo de álcool pelo seu médico de família no decorrer da consulta; analisar factores associados (idade, escolaridade, consumo de álcool) às atitudes encontradas.

Tipo de estudo: Observacional, transversal e analítico.

Local: Agrupamentos de Centros de Saúde I, III e VI da área da grande Lisboa.

População: Utentes de ambos os sexos, com idade igual ou superior a 16 anos, letrados, que frequentam os CSP.

Métodos: A uma amostra de conveniência de 175 utentes dos CSP da área da grande Lisboa, após recolha de informação demográfica, foi aplicado um questionário sobre atitudes em relação ao rastreio do consumo nocivo de álcool e realizado um rastreio de consumo nocivo de álcool (AUDIT-C). Foram utilizados os testes do $\chi^{2}$ e $t$ de Student, considerando um nível de significância de 0,05.

Resultados: A maioria dos utentes (mais de $80 \%$ ) demonstrou apoio ao rastreio dos consumos de álcool por parte do seu médico. A resposta a 4 de 10 afirmações sobre atitudes quanto a este rastreio mostrou-se relacionada com a idade e escolaridade. Contabilizaram-se 41 indivíduos (23,4\%) com rastreio AUDIT-C positivo, representando 38,5\% dos homens e 14,5\% das mulheres.

Conclusão: Os utentes apoiam o rastreio sobre consumos de álcool pelo seu médico, independentemente de quaisquer variáveis ou do método de rastreio, estando igualmente abertos ao aconselhamento sobre esses consumos.

Palavras-chave: Alcoolismo; Consumo de álcool; Rastreio; Cuidados de saúde primários.

\section{INTRODUÇÃO}

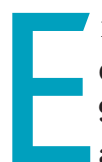
m Portugal, o consumo de álcool é dos mais elevados do mundo, tendo-se situado, no ano 2003, em 9,6 litros de álcool puro per capita, ${ }^{1}$ tornando-nos assim o oitavo consumidor a nível mundial. Desta forma, a alcoolização da sociedade portuguesa é uma realidade. De facto, de acordo com estudos de Balsa et al., entre 2001 e 2007 a prevalência do consumo de bebidas alcoólicas aumentou $3,5 \%$, de $75,6 \%$ para $79,1 \%$, referindo $38,5 \%$ dos jovens entre os 20 e os 24 anos e $34,6 \%$ dos jovens dos jovens entre os 15 e os 19 anos embriaguez pelo menos uma vez (2,8\% dos jovens dos 20 aos 24 anos e $1,2 \%$ dos jovens dos 15 aos 19 anos embriagou-se dez ou mais vezes). ${ }^{2}$

Deste modo é cada vez maior o ênfase dado, a nível nacional, ${ }^{3,4}$ ao papel dos Cuidados de Saúde Primários (CSP) enquanto estruturas de proximidade, tendo em vista uma detecção e sinalização precoce de indivíduos em risco.

*Faculdade de Medicina da Universidade de Lisboa

**Professora auxiliar da Faculdade de Medicina de Lisboa, médica de família, assessora do Instituto da Droga e da Toxicodependência para os problemas ligados ao álcool.
Para auxiliar os clínicos nesta tarefa foram desenvolvidos vários instrumentos que permitem um rastreio sistemático. Entre estes instrumentos encontram-se vários questionários (CAGE, AUDIT-C, T-ACE, TWEAK, CRAFFT) que, com uma sensibilidade entre os 69 e os $95 \%$ e uma especificidade que varia entre os 71 e os $90 \%,{ }^{5,6}$ permitem o rastreio de uma larga maioria da população, indicando a presença de grupos com possíveis consumos de alto-risco, abuso e dependência.

Os estudos sobre instrumentos de rastreio nos CSP concentram-se num amplo espectro de consumos, incluindo situações de risco, consumo nocivo/abuso e dependência de álcool. O consumo de risco é geralmente definido através do estabelecimento de um limiar de consumo deálcool e é também referido como consumo problemático ou excessivo. Neste padrão de consumo encontram-se os utentes em risco de apresentar consequências relacionadas com o consumo de álcool, quer através da quantidade consumida, quer devido ao efeito do álcool sobre outras comorbilidades. Os consumidores nocivos apresentam danos físicos ou psicológicos secundários ao consumo de álcool. 
Pacientes com abuso de álcool e dependência experienciam de forma marcada e repetida efeitos físicos e sociais negativos, incluindo um desejo intenso, descontrolo sobre o uso, continuação dos consumos independentemente das consequências, alta prioridade dada aos consumos em detrimento de outras actividades e obrigações, aumento da tolerância ao álcool e sintomas de privação quando o consumo é descontinuado. ${ }^{7}$ Estes esquemas de classificação são habitualmente usados para estratificar os utentes com relação ao prognóstico, gravidade e regimes de tratamento apropriado.

No entanto, vários estudos referem que ao nível dos CSP o rastreio se encontra em níveis bastante inferiores ao desejado. ${ }^{9,10,13}$ Nos Estados Unidos da América um estudo demonstrou que, apesar de $88 \%$ dos médicos referirem realizar um rastreio ao consumo de álcool por parte dos seus doentes, apenas $13 \%$ utilizava um questionário formal de rastreio e, mesmo nos indivíduos com rastreio positivo, uma correcta abordagem e referenciação não era realizada. ${ }^{5}$ A utilização de meios de diagnóstico laboratorial (marcadores bioquímicos como a gama-glutamiltranspeptidase, o volume globular médio ou a transferrina deficiente em hidratos de carbono) apresentam uma utilização ainda mais reduzida. ${ }^{6}$

Esta reduzida utilização dos instrumentos de rastreio é justificada pelos clínicos por: conhecimento e capacidades insuficientes para lidar com o problema, noções pessimistas acerca da utilidade do rastreio e receio que os seus doentes se sintam ofendidos com as questões e que isso, consequentemente, acabe por fragilizar a relação médico-doente. ${ }^{10-12}$ No entanto, estudos realizados a nível internacional parecem não acompanhar os receios dos clínicos, demonstrando uma grande abertura dos doentes em relação ao tema. ${ }^{13,14} \mathrm{Em}$ Portugal desconhece-se a receptividade em relação à abordagem deste tipo de questões em consulta.

Outro dos grandes entraves apontados pelos médicos dos CSP a uma mais ampla utilização dos questionários de rastreio é o tempo reduzido de que dispõem para cada consulta e a multiplicidade de tarefas que se impõem a um médico generalista. ${ }^{10,15}$

Assim, são objectivos deste trabalho:

- Caracterizar o consumo de álcool dos utentes dos Cuidados de Saúde Primários (CSP) com idade igual ou superior a 16 anos;

- Determinar as atitudes dos participantes face ao rastreio do consumo de álcool pelo seu médico de família no decorrer da consulta;

- Analisar factores associados (idade, escolaridade, consumo de álcool) às atitudes encontradas.

\section{MÉTODOS}

A população participante do estudo foram os utentes dos Agrupamentos de Centros de Saúde I, III eVI da região da grande Lisboa, letrados e com idade igual ou superior a 16 anos. A amostragem foi de conveniência, sendo solicitado aos utentes, com idade igual ou superior a 16 anos, com consulta marcada durante os 8 dias (um por semana, com rotação no dia) de Janeiro e Fevereiro de 2010 em que decorreu o estudo, o preenchimento, a título voluntário, de um inquérito de opinião para investigação científica. Os participantes preencheram o questionário de forma anónima (não tendo sido utilizados identificadores de qualquer tipo) enquanto aguardavam pela consulta médica. O tempo de preenchimento do questionário foi cerca de cinco minutos.

O questionário referido, usado como instrumento de medida, foi uma folha de registo anónima, inspirada nos trabalhos de Miller,,$^{13,14}$ traduzida pelo autor, constituída por 10 itens, de auto-preenchimento pelos participantes e que abordava as suas atitudes em relação ao consumo de álcool, focando as seguintes temáticas: 1) Atitude acerca da adequação de questões de rastreio alcoólico; 2) Abertura ao rastreio bioquímico do consumo de álcool; 3) Reacção emocional ao rastreio; 4) Abertura ao aconselhamento com vista à redução de consumos nocivos; 5) Honestidade na resposta a questões de rastreio (ver Quadro IV). As frases/opiniões foram classificadas, de acordo com o questionário de Miller, através de uma escala de Likert de cinco pontos (concordo totalmente, concordo, sem opinião, discordo, discordo totalmente).

As outras variáveis em estudo foram: género, idade (anos) e escolaridade (4, 9, 12 e mais que 12 anos).

De seguida, foi aplicada uma ferramenta de rastreio ao consumo nocivo de álcool, o AUDIT-C, composto pelas três primeiras questões do rastreio AUDIT (questionário aplicado internacionalmente e validado para a língua portuguesa, constituído por dez questões que avaliam o consumo nocivo de álcool, sintomas de dependência e consequências nocivas da utilização abusiva de álcool). ${ }^{16} \mathrm{~A}$ pontuação AUDIT-C varia de 0 a 12 pontos, considerando-se o rastreio positivo se a pontuação obtida for igual ou superior a quatro pontos (para um nível de sensibilidade entre os 91 e os $100 \%$ e uma especificidade que varia dos 53 aos $68 \%$ ). ${ }^{17}$

Fez-se um estudo estatístico sequencial dos dados recolhidos utilizando o softwareSPSS v.17 para Windows. Foi realizada primeiramente uma caracterização da população em estudo, tendo em seguida sido realizados dois conjuntos de análises, recorrendo ao teste $t$ de Student (no caso de variável contínua) e ao $\chi^{2}$ (restantes situações). Inicialmente foi efectuada uma análise de frequências das res- 
postas obtidas que revelou uma distribuição não normal das respostas a cada uma das dez afirmações, sendo que a maioria dos participantes respondeu concordo ou concordo totalmente ou, no caso de afirmações negativas, discordo ou discordo totalmente. Posteriormente realizou-se uma recodificação das respostas obtidas para apenas duas variáveis dicotómicas: concordante (concordo totalmente e concordo) e não concordante (sem opinião, discordo e discordo totalmente). Para aquelas afirmações em que a discordância reflecte uma opinião favorável acerca do rastreio (por ex: «a quantidade de álcool que bebo é pessoal e confidencial e o meu médico não devia questionar-me acerca disso») para a análise estatística combinaram-se as respostas discordo totalmente e discordo.

A proporção de participantes com respostas concordantes com o rastreio, com base na recodificação realizada, foi considerada a variável dicotómica dependente na análise estatística efectuada para avaliar o efeito dos factores demográficos nas respostas obtidas. O valor $p$ foi considerado significativo se inferior a 0,05.

A recolha de dados decorreu de acordo com os princípios estabelecidos na declaração de Helsínquia e o relatório de investigação obteve, a posteriori, parecer favorável da Comissão de Ética do Hospital de Santa Maria/Faculdade de Medicina da Universidade de Lisboa, em Lisboa.

\section{RESULTADOS}

Um total de 224 utentes tinham consulta durante o período do estudo e foram convidados a preencher o questionário. Destes, 30 recusaram e 13 não cumpriam os critérios de inclusão. Desta forma o questionário foi aplicado a 181 utentes. Destes, 6 foram anulados, 4 por não se apresentarem completamente preenchidos e 2 após desistência do participante. Desta forma, os resultados são baseados numa amostra total de 175 utentes.

Como referido no Quadro I, a amostra era constituída maioritariamente por mulheres (62,9\%) e a idade média era de 49,7 anos (desvio-padrão 18,7 anos, mediana de 51 anos, variando as idades entre os 16 e os 86 anos). Dos indivíduos participantes, $41,7 \%$ apresentavam um nível de escolaridade igual ou inferior a nove anos e $71,4 \%$ igual ou inferior a doze anos de ensino.

Dos questionários obtidos, 16 mulheres (14,5\%) e 25 homens $(38,5 \%)$ apresentaram um rastreio AUDIT-C positivo, totalizando 41 indivíduos $(23,4 \%)$ com um rastreio AUDIT-Cigual ou superior a quatro pontos, indicador de possível consumo nocivo.

O Quadro II apresenta as questões AUDIT-C com a proporção de respostas positivas e negativas a cada pergunta, onde se verifica que $73,7 \%$ dos indivíduos apresenta um consumo habitual de álcool. Destaca-se o facto de $27,4 \%$ dos indivíduos referirem consumos tipo binge, uma proporção superior aos 10,3\% que referem consumo habitual de bebidas alcoólicas.

O Quadro III compara os dados sócio-demográficos de acordo com a pontuação AUDIT-C. A aplicação do teste $\chi^{2} \mathrm{e}$ do teste $t$ de Student revelou uma associação estatisticamente significativa entre o consumo nocivo de álcool e o género masculino $\left(\chi^{2}=13,03 ; p=0,001\right)$.

A maioria dos pacientes expressou opinião francamente positiva em relação ao rastreio do consumo de álcool por parte do seu médico assistente. O Quadro IV mostra a proporção de concordância/discordância com cada afirmação.

É importante salientar que a menor taxa de concordância/discordância foi obtida com a afirmação «Ficaria incomodado se o meu médico me perguntasse a quantidade de álcool que eu bebo» com $60 \%$ dos participantes a discordar da afirmação. Contudo, de um modo geral, a maioria dos questionados ( $>80 \%$ ) eram favoráveis e receptivos ao rastreio do consumo nocivo de álcool.

Cada afirmação foi analisada com cada um de quatro factores: género, idade, escolaridade e score AUDIT-C, verificando-se que em 6 das 10 afirmações as respostas dadas não foram significativamente influenciadas por nenhuma das variáveis estudadas. As restantes quatro afirmações demonstraram a presença de relações significativas entre a resposta dada e a demografia do utente.

Desta forma, em relação à afirmação «Ficaria incomodado se o meu médico me perguntasse a quantidade de álcool que bebo», verificou-se uma associação estatisticamente significativa entre a resposta e escolaridade $\left(\chi^{2}=\right.$

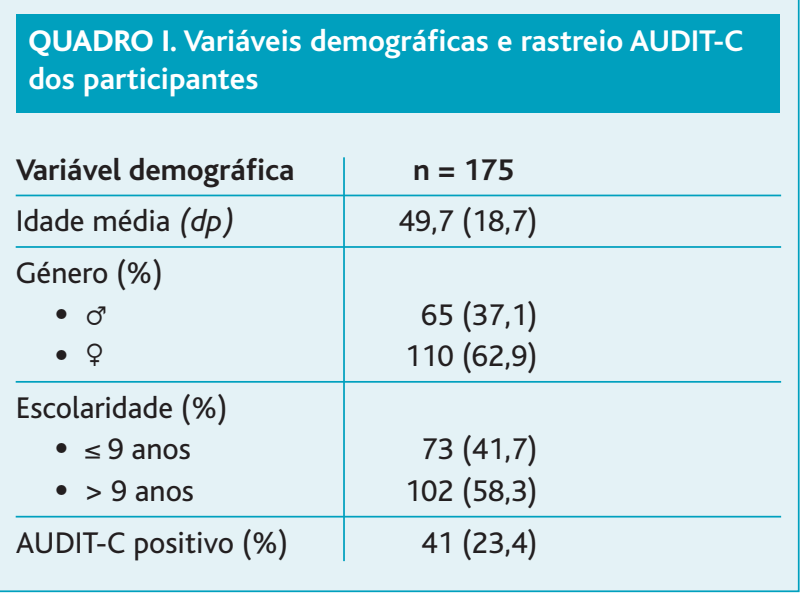

$d p$ - Desvio-padrão 
$11,42 ; p=0,001)$ e/ou idade $\left(\chi^{2}=7,34 ; p=0,025\right)$. Especificamente os utentes mais jovens ou com maior escolaridade têm maior probabilidade de discordar da afirmação.

No que se refere às afirmações «A quantidade de álcool que bebo é pessoal e confidencial e o meu médico não devia questionar-me acerca disso» e «Ficaria aborrecido se o meu médico me perguntasse a quantidade deálcool que eu bebo», foram identificadas como factores significativamente influentes na resposta obtida a escolaridade $\left(\chi^{2}=\right.$ $\left.12,98, p=0,001 \mathrm{e} \chi^{2}=18,45, p=0,001\right)$ e a idade $\left(\chi^{2}=8,60, p\right.$ $\left.=0,014 \mathrm{e} \chi^{2}=6,468, p=0,039\right)$ respectivamente. Desta forma os utentes mais jovens ou com maior nível de escolaridade tem uma maior tendência a discordar das afirmações.

Em relação à afirmação «Se o meu médico me perguntasse quanto álcool eu bebo, provavelmente não lhe daria uma resposta honesta", foram identificados como factores estatisticamente influentes na resposta obtida a escolaridade $\left(\chi^{2}=6,43 ; p=0,011\right)$, a idade $\left(\chi^{2}=6,33 ; p=0,042\right)$ e o score AUDIT-C $\left(\chi^{2}=3,31 ; p=0,069\right)$. Assim, um maior nível de escolaridade, uma menor idade ou um score AUDIT-C negativo conduzem a uma maior probabilidade de discordar da afirmação.

Torna-se importante salientar que, apesar destes resultados estatisticamente significativos, a maioria dos utentos de ambos os grupos apresentou receptividade ao rastreio de consumos nocivos de álcool.

\section{DISCUSSÃO}

Os resultados acima descritos não apoiam o receio de muitos clínicos de que os seus doentes irão rejeitar ou melindrar-se com a realização de rastreio e aconselhamento sobre consumos etanólicos nocivos. A análise efectuada mostra mesmo que mais de 80\% dos indivíduos estão receptivos ao rastreio (quer através de questionário, quer bioquímico) e ao aconselhamento sobre consumos nocivos.

É igualmente possível afirmar que os dados demográficos e o score AUDIT-C não são preditivos da opinião dos utentes acerca do rastreio do consumo nocivo de álcool. Os doentes em risco de desenvolver problemas ligados ao álcool não apresentam respostas diferentes quanto à receptividade em relação ao rastreio de consumos nocivos de álcool, apoiando assim a presença universal do rastreio na prática clínica.

A proporção de indivíduos com o rastreio positivo é de $23,4 \%$, um valor que está de acordo com os resultados obtidos em outros trabalhos, quer a nível nacional, ${ }^{18}$ quer a nível internacional. ${ }^{4,13-14}$ No

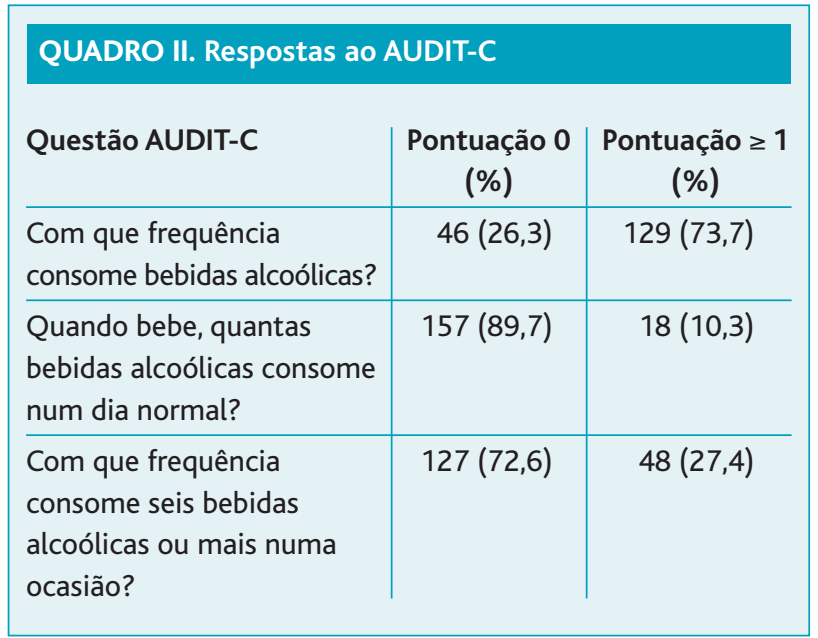

entanto, este valor pode estar subestimado, pois começam a aparecer evidências de que a sensibilidade do rastreio diminui com o aumento da faixa etária da população analisada, em virtude das alterações associadas ao envelhecimento, ${ }^{19,20}$ de que são exemplo a diminuição da função hepática e dos níveis de álcool desidrogenase. O que faz com que, apesar de tendencialmente existir um menor consumo nas faixas etárias mais avançadas, a concentração de álcool no sangue pode ser superior à de populações mais jovens. ${ }^{21,22}$ Tendo em conta que a faixa de população em maior crescimento em todo o mundo é a das pessoas com mais de 65 anos, uma reavaliação e construção de rastreios

\begin{tabular}{|c|c|c|c|}
\hline \multicolumn{4}{|c|}{$\begin{array}{l}\text { QUADRO III. Variáveis demográficas para indivíduos AUDIT-C } \\
\text { positivo e negativo }\end{array}$} \\
\hline Variável & $\begin{array}{c}\text { AUDIT-C } \\
\text { Negativo }(n=134)\end{array}$ & $\begin{array}{c}\text { AUDIT-C } \\
\text { Positivo }(n=41)\end{array}$ & Valor $p$ \\
\hline Idade média ( $d p)$ & $48(18,7)$ & $55(18,1)$ & NS $(0,36)$ \\
\hline - $16-30(\%)$ & $26(19,4)$ & $4(9,8)$ & \\
\hline - $30-65(\%)$ & $79(59,0)$ & $24(58,5)$ & NS $(0,22)$ \\
\hline - > $65(\%)$ & $29(21,6)$ & $13(31,7)$ & \\
\hline Género & & & 0,001 \\
\hline - Masculino (\%) & $40(61,5)$ & $25(38,5)$ & \\
\hline - Feminino (\%) & $94(85,5)$ & $16(14,5)$ & \\
\hline \multicolumn{4}{|l|}{ Escolaridade (\%) } \\
\hline - $\leq 9$ anos & $53(72,6)$ & $20(27,4)$ & \\
\hline - > 9 anos & $81(79,4)$ & $21(20,6)$ & NS $(0,6)$ \\
\hline
\end{tabular}

NS - Valor Não Significativo; $d p$ - Desvio-padrão 


\begin{tabular}{|c|c|c|c|}
\hline Afirmação & Resposta favorita & (\%) & Valor $p$ \\
\hline $\begin{array}{l}\text { Como parte da avaliação da minha saúde, o meu médico deve sentir-se } \\
\text { à vontade para perguntar a quantidade de álcool que eu bebo }\end{array}$ & $\begin{array}{l}\text { Concordo/Concordo } \\
\text { Totalmente }\end{array}$ & 96,0 & NS \\
\hline $\begin{array}{l}\text { Ficaria incomodado se o meu médico me perguntasse a quantidade de } \\
\text { álcool que eu bebo }\end{array}$ & $\begin{array}{l}\text { Discordo/Discordo } \\
\text { Totalmente }\end{array}$ & 60,0 & $\begin{array}{r}\text { Escolaridade }(p=0,001) * \\
\text { Idade }(p=0,025) *\end{array}$ \\
\hline $\begin{array}{l}\text { Se o facto de beber estiver a afectar a minha saúde (como a tensão } \\
\text { arterial), o meu médico deve aconselhar-me a reduzir o consumo de álcool }\end{array}$ & $\begin{array}{l}\text { Concordo/Concordo } \\
\text { Totalmente }\end{array}$ & 97,1 & NS \\
\hline $\begin{array}{l}\text { Se o meu médico sugerisse a realização de uma análise que dissesse se } \\
\text { estou a beber em excesso para a minha saúde, gostaria de ser testado }\end{array}$ & $\begin{array}{l}\text { Concordo/Concordo } \\
\text { Totalmente }\end{array}$ & 96,6 & NS \\
\hline $\begin{array}{l}\text { Se o meu médico me perguntasse quanto é que eu bebo, responderia } \\
\text { honestamente }\end{array}$ & $\begin{array}{l}\text { Concordo/Concordo } \\
\text { Totalmente }\end{array}$ & 94,9 & NS \\
\hline $\begin{array}{l}\text { Se o facto de beber estiver a afectar a minha saúde (como a tensão } \\
\text { arterial), o meu médico deve aconselhar-me a parar de beber }\end{array}$ & $\begin{array}{l}\text { Concordo/Concordo } \\
\text { Toalmente }\end{array}$ & 95,4 & \\
\hline $\begin{array}{l}\text { A quantidade de álcool que bebo é pessoal e confidencial e o meu } \\
\text { médico não devia questionar-me acerca disso }\end{array}$ & $\begin{array}{l}\text { Discordo/Discordo } \\
\text { Totalmente }\end{array}$ & 80,0 & $\begin{array}{r}\text { Idade }(p=0,014) \\
\text { Escolaridade }(p=0,001)\end{array}$ \\
\hline $\begin{array}{l}\text { Se o meu médico pensar que o álcool afecta a minha saúde, deve } \\
\text { sentir-se à vontade para pedir uma análise sanguínea para saber se estou } \\
\text { a beber demais }\end{array}$ & $\begin{array}{l}\text { Concordo/Concordo } \\
\text { Totalmente }\end{array}$ & 93,7 & NS \\
\hline $\begin{array}{l}\text { Ficaria aborrecido se o meu médico me perguntasse a quantidade de } \\
\text { álcool que eu bebo }\end{array}$ & $\begin{array}{l}\text { Discordo/Discordo } \\
\text { Totalmente }\end{array}$ & 78,3 & $\begin{array}{r}\text { Idade }(p=0,039) \\
\text { Escolaridade }(p=0,001)\end{array}$ \\
\hline $\begin{array}{l}\text { Se o meu médico me perguntasse quanto álcool eu bebo, provavelmente } \\
\text { não lhe daria uma resposta honesta }\end{array}$ & $\begin{array}{l}\text { Discordo/Discordo } \\
\text { Totalmente }\end{array}$ & 74,3 & $\begin{array}{r}\text { Idade }(p=0,042) \\
\text { Escolaridade }(p=0,011) \\
\text { AUDIT-C positivo }(p=0,07)\end{array}$ \\
\hline
\end{tabular}

NS - Valor Não Significativo para todas as variáveis estudadas:*

específicos deve ser ponderada, acrescendo às razões apontadas anteriormente o facto de este estudo ter apurado uma taxa de $31,7 \%$ de rastreios positivos para consumos nocivos no escalão etário acima dos 65 anos.

Na análise das respostas ao rastreio AUDIT-C destaca-se o facto de $27,4 \%$ dos indivíduos referirem pelo menos um consumo tipo binge, enquanto apenas $10,3 \%$ referem consumos superiores a duas bedidas por dia. Estes resultados apontam para um consumo de álcool mais concentrado no tempo que até recentemente não era valorizado na literatura, não se encontrando indicadores para avaliar esta realidade nos principais estudos epidemiológicos nacionais, como os Inquéritos Nacionais de Saúde. ${ }^{18}$ Esta realidade tradicionalmente encontra-se associada aos indivíduos mais jovens e a países do norte da Europa. Esta problemática necessita de mais investigação no sentido de avaliar uma possível alteração no padrão de consumo de álcool.

A principal diferença estatisticamente significativa encontrada entre os grupos AUDIT-C positivo e negativo está relacionada com uma maior proporção de homens no subgrupo AUDIT-C positivo (38,5\%). Este facto parece sugerir a manutenção de um padrão de consumo alcoólico individual tipicamente masculino.

Na prática clínica diária, a maioria dos rastreios positivos será constituída por consumidores de risco, sem critérios para o diagnóstico de consumo nocivo/abuso ou dependência de álcool. No entanto, isto não significa que nada deva ser feito, sendo importante o aconselhamento e, se possível, a realização de intervenção breve tendo em vista a redução do consumo. ${ }^{23}$

As variáveis demográficas investigadas e o status AUDIT de uma forma geral não estavam relacionados com a atitu- 
de em relação ao rastreio, demonstrando todos os subgrupos analisados, sem diferença estatística significativa, uma opinião bastante favorável sobre todas as temáticas abordadas.

Contudo, a idade e a escolaridade foram associadas à reacção emocional ao rastreio, atitude acerca da adequação de questões de rastreio alcoólico e honestidade na resposta a questões de rastreio, sendo que os indivíduos mais jovens e/ou academicamente mais diferenciados apresentavam maior abertura para o rastreio. Tal poderá dever-se a uma maior liberalidade e desmistificação do conceito de abuso de substâncias, assim como a uma noção mais holística da saúde, em que esta está dependente dos estilos de vida adoptados. Desta forma, os utentes mais jovens e/ou academicamente mais diferenciados parecem mais disponíveis para uma entrevista clínica abrangente, integrada num conceito de Medicina mais preventiva, enquanto a população mais idosa e/ou menos diferenciada tende a valorizar uma Medicina mais dirigida e essencialmente curativa, centrada no médico.

De salientar que o efeito das variáveis idade e escolaridade está ausente no caso do rastreio bioquímico do consumo de álcool, onde se verificou que mais de $90 \%$ dos indivíduos eram favoráveis a esta forma de rastreio. Os utentes AUDIT-C positivos não apresentam uma menor probabilidade de adesão a este tipo de rastreio. Mais estudos para avaliar esta situação devem ser realizados.

É também importante notar que o score AUDIT-C não influencia as respostas obtidas. Este facto deve ser salientado pois um dos receios expressos pelos clínicos dos CSP é o facto de se considerar que os pacientes com possíveis consumos nocivos não estão disponíveis para abordar o problema ou aceitar aconselhamento por parte do seu médico assistente. ${ }^{8,10}$

A questão na qual o AUDIT se encontra mais próximo da significância estatística ( $\mathrm{p}=0,07$ ) é uma questão que aborda a honestidade das respostas dadas. Tal pode ser atribuído à noção do indivíduo de que apresenta um consumo exagerado que poderá ser problemático, mas que não deseja ou não se sente preparado para discutir no âmbito da actual consulta.

A diferença entre as questões «Se o meu médico me perguntasse quanto é que eu bebo, responderia honestamente» $\mathrm{e}$ «Se o meu médico me perguntasse quanto é que eu bebo, provavelmente não lhe daria uma resposta honesta» (94,9\% concordando vs 74,3\% discordando), pode ser entendida, pelo menos parcialmente, como consequência do efeito de Hawthorn (tendência dos participantes de realizar aquilo que pensam ser esperado deles), no entanto, ten- do em conta o nível de literacia da amostra, uma interpretação incorrecta da afirmação não pode ser excluída.

São limitações importantes do presente estudo o tratar-se de uma população exclusivamente urbana, o tipo de amostragem de conveniência (utentes presentes nos centros de saúde e predominantemente no período da tarde) e o tamanho reduzido da amostra, aceitando-se no entanto esta metodologia pelo facto de se tratar de um estudo exploratório.

Outras limitações do estudo são a utilização de escalas e classificações cuja validade não foi analisada previamente para a população em estudo.

Ainda, a realização do questionário nas salas de espera dos centros de saúde participantes e o facto de o entrevistador ser um estudante de Medicina introduz um viés, relacionado com as atitudes socialmente esperadas.

Sugere-se futuramente a realização de um estudo de âmbito nacional, em que a dimensão amostral e respectiva técnica fossem determinadas de forma a obter uma amostra representativa. Seria também mais adequada a entrevista com recurso a chamada telefónica, previamente notificada mediante aviso postal. A colheita dos dados deveria ser realizada em pelo menos quatro meses correspondentes a diferentes períodos do ano, com vista à não interferência nos resultados de potenciais variações/alterações cíclicas nos padrões de consumo.

Pelo exposto acima não é possível uma generalização dos resultados obtidos, que no entanto se apresentam de uma forma geral semelhantes a outros estudos a nível internacional. $^{13,14}$

Os resultados deste trabalho mostram uma grande abertura dos pacientes em relação ao rastreio e aconselhamento, independentemente da quantidade de álcool que consomem e de outras variáveis sociodemográficas, esvaziando assim os argumentos dos que afirmam que os utentes se podem sentir ofendidos por estas perguntas. Este estudo encontrou, através de uma simples ferramenta de rastreio, o AUDIT-C, uma prevalência provável de consumo alcoólico nocivo de $23,4 \%$, entre os utentes dos CSP. Desta forma, e tendo em conta a facilidade com que este rastreio pode ser realizado, sugere-se a aplicação anual deste rastreio por parte dos clínicos (como recomendado pela Organização Mundial de Saúde). ${ }^{24,25}$

\footnotetext{
REFERÊNCIAS BIBLIOGRÁFICAS

1. World alcohol consumption 2005. In:World Drinking Trends. London:World Advertising Research Council; 2005.

2. Balsa C, Vital C, Urbano C, Pascueiro L. Inquérito Nacional ao Consumo de Substâncias Psicoactivas na População Geral: Portugal 2007. Lisboa: CEOS/FCSH/UNL; 2008.

3. Colom J, Segura L, Gual A. Alcohol y atención primaria de salud: el proyecto PHEPA. Rev Port Clin Geral2008 Mar-Abr; 24 (2): 283-5.

4. Ribeiro,C. Impactos do álcool e estratégias de intervenção na Europa. Que papel para
} 
os Cuidados de Saúde Primários? Rev Port Clin Geral 2008 Mar-Abr; 24 (2): 323-9.

5. Cherpitel CJ.Analysis of cut points for screening instruments for alcohol problems in the emergency room. Journal Stud Alcohol 1995 Nov; 56 (6): 695-700.

6. Burns E, Gray R, Smith LA. Brief screening questionnaires to identify problem drinking during pregnancy: a systematic review. Addiction 2010 Apr; 105 (4): 601-14

7. WHO Regional Office for Europe. European Alcohol Action Plan. Copenhaga: OMS; 1992.

8. Friedmann PD, McCullough D, Chin MH, Saitz R. Screening and intervention for alcohol problems: a national survey of primary care physicians and psychiatrists. J Gen Intern Med 2000 Feb; 15 (2): 84-91.

9. Miller PM, Ornstein SM, Nietert PJ,Anton RF. Self-report and biomarker alcohol screening by primary care physicians: the need to translate research into guidelines and practice. Alcohol Alcohol 2004 Jul-Aug; 39 (4): 325-8.

10. Kääriäinen J, Sillanaukee P, Poutanen P, Seppä K. Opinions on alcohol-related issues among professionals in primary, occupational and specialized health care. Alcohol Alcohol 2001 Mar-Apr; 36 (2): 141-6.

11. Ribeiro C. Álcool: impactos no individuo e na sociedade - que papel para os cuidados de saúde primários? Rev Port Clin Geral 2008 Mar-Abr; 24 (2): 269-74.

12. Beich A., Gannik D., Malterud K. Screening and brief intervention for excessive alcohol use: qualitative interview study of the experiences of general practitioners. BMJ 2002 Oct 19; 325 (7369): 870-2.

13. Miller PM, Thomas SE, Mallin R. Patient attitudes towards self-report and biomarker alcohol screening by primary care physicians. Alcohol Alcohol 2006 May-Jun; 41 (3): 306-10.

14. Miller PM, Ravenel MC, Shealy AE, Thomas S. Alcohol screening in dental patients: The prevalence of hazardous drinking and patients' attitudes about screening and advice. J Am Dent Assoc 2006 Dec; 137 (12): 1692-8.

15. Kaner EF, Heather N, McAvoy BR, Lock CA, Gilvarry E. Intervention for excessive alcohol consumption in primary health care: attitudes and practices of English general practitioners. Alcohol Alcohol 1999 Jul-Aug; 34 (4): 559-66.

16. Smith PC, Schmidt SM, Allensworth-Davies D, Saitz R. Primary care validation of a single-question alcohol screening test. J Gen Intern Med 2009 Jul; 24 (7):783-8.

17. Gual A, Segura L, Contel M, Heather N, Colom J. AUDIT-3 and AUDIT-4: effectiveness of two short forms of the alcohol use disorders identification test. Alcohol Alcohol
2002 Nov-Dec; 37 (6): 591-6

18. Inquérito Nacional de Saúde 2005/2006. Lisboa: Instituto Nacional de Estatística / INSA; 2009. p. 66-8.

19. Zimniak P. Detoxification reactions: relevance to aging. Ageing Res Rev 2008 Dec; 7 (4): 281-300

20. Moos RH, Schutte KK, Brennan PL, Moos BS. Older adults' alcohol consumption and late-life drinking problems: a 20-year perspective. Addiction 2009 Aug; 104 (8): 1293 302.

21. National Health Interview Survey. Family core and sample adult questionnaires, 2003. Disponível em: http://www.cdc.gov/nchs/data/hus/tables/2003/hus065.pdf [acedido a 24/10/2011].

22. Grant BF, Dawson DA, Stinson FS, Chou SP, Dufour MC, Pickering RP. The 12-month prevalence and trends in DSM-IV alcohol abuse and dependence: United States, 1991-1992 and 2001-2002. Drug Alcohol Depend 2004 Jun 11; 74 (3): 223-34.

23. Saitz R. Clinical practice: unhealthy alcohol use. N Engl J Med 2005 Feb 10; 352 (6): 596-607.

24. Babor TF, Higgins-Biddle JC, Saunders JB, Monteiro MG. A U D IT - The Alcohol Use Disorders Identification Test: Guidelines for Use in Primary Care. 2nd ed. Geneva:WHO; 2001.

25. Fiellin D., Reid MC, O'Connor PG. Screening for alcohol problems in primary care: a systematic review. Arch Intern Med 2000 Jul 10; 160: 1977-89.

\section{CONFLITOS DE INTERESSE}

Os autores declaram não ter conflitos de interesse.

\section{ENDEREÇO PARA CORRESPONDÊNCIA}

Pedro Pereira Campos

Rua Barbosa du Bocage Lt. 33 R/C Esq

2620-214 Ramada

E-mail: pmcampos3676@gmail.com

Recebido em 17/06/2011

Aceite para publicação em 26/02/2012

\section{ABSTRACT}

\section{PATIENT ATTITUDES TO SCREENING OF ALCOHOL CONSUMPTION IN PRIMARY HEALTH CARE}

Objectives: To characterize the alcohol consumption of patients in Primary Health Care, to determine the attitude of patients towards screening for harmful alcohol consumption by their physician, to determine if these attitudes are related to the patient's AUDIT-C status, and to analyze factors (age, education, alcohol consumption) related to attitudes towards alcohol screening conducted by the family physician during the consultation.

Study design: Analytical, cross sectional

Setting: Health Centre Groups I, III and VI of the Greater Lisbon area.

Participants: Patients of both genders, aged 16 years or older.

Methods: A convenience sample of 175 patients in primary health care in the Lisbon area was used. After collecting demographic information from patients, we administered a questionnaire on attitudes towards the screening of harmful alcohol consumption and the AUDIT-C instrument. The chi-square and Student t-test were used, with significance set at the 0,05 level.

Results: Most patients (> 80\%) expressed support for the screening of alcohol intake by their physician. The answer to four of the statements was related to age and educational level. Forty-one individuals (23.4\%) with AUDIT-C positive screening were found, representing $38.5 \%$ of men and $14.5 \%$ of the women.

Conclusions: Patients accept screening of alcohol consumption by their physician, independent of demographic variables or the screening method used. Patients are also open to advice regarding their alcohol intake.

Key Words: Alcoholism; Alcohol drinking; Screening; Primary health care. 